\title{
Effect of Xiaochaihu Decoction on Cytokines and MAO in Experimental Autoimmune Prostatitis Rats
}

\author{
Wang Zhiqiang ${ }^{1}$ Zhu Liping $^{2}$ Mu Zhenyu ${ }^{3}$ Wang Xiaogang4* \\ ${ }^{I}$ Department of Urology, Shouguang Hospital of Traditional Chinese Medicine, Shouguang, 262700, China, \\ https://orcid.org/0000-0003-1459-6602. ${ }^{2}$ Department of Oncology, Shouguang Hospital of Traditional Chinese \\ Medicine, Shouguang, 262700, China, https://orcid.org/0000-0002-2983-8945. ${ }^{3}$ Department of Neurology, Shouguang \\ Hospital of Traditional Chinese Medicine, Shouguang, 262700, China, https://orcid.org/0000-0002-5782-8795. \\ ${ }^{4 *}$ Department of Pharmacy, Shouguang Hospital of Traditional Chinese Medicine, Shouguang, 262700, China, \\ SGSwangxiaogang@163.com, https://orcid.org/0000-0003-2088-0685.
}

Article History Received 15 August 2020 Accepted 25 September 2020 Published 31 December 2020 Cite this Article Wang Zhiqiang, Zhu Liping, Mu Zhenyu, Wang Xiaogang. Effect of Xiaochaihu Decoction on cytokines and MAO in experimental autoimmune prostatitis rats [J].Medical Research, 2020.2(4):10-15, http://dx.doi.org/10.6913/MRHK.202012_2(4).0002

Copyright @ 2020 Creative Publishing Co., Limited. All rights reserved. Email:mrhk26640333@gmail.com.

\section{ABSTRACT}

Objective To observe the effect of Xiaochaihu Decoction on cytokines and MAO in model rats with experimental autoimmune prostatitis (EAP) to provide an experimental basis for the use of Chinese herbal prescriptions in the treatment of chronic prostatitis.

Methods Thirty male Wistar rats were randomly divided into blank Group, model group and treatment group (Xiaochaihu Decoction). Except the blank group, rats in model and treatment groups were injected subcutaneously in multiple points on days 0 and 30 with prostatic protein extractive solution $(60 \mathrm{mg} / \mathrm{mL})$, and intraperitoneally injected with diph-theria-pertussis and tetanus vaccine (DPT vaccine) to establish the EAP model. Model rats were administrated Xiaochaihu Decoction and were sacrificed after 4 weeks. Pathological changes in the prostate gland were observed with HE staining and changes in serum interleukin-6 (IL-6), interleukin-8 (IL-8), and Tumor necrosis factor- $\alpha$ (TNF- $\alpha$ ) levels were detected with enzyme-linked immunosorbent assay (ELISA). Quantitative PCR to observe the expression of IL-17, CCL2 and MAOA/B.

Results After treatment with Xiaochaihu Decoction, the serum IL-6, IL-8, and TNF- $\alpha$ levels all significantly lower in the treatment group as compared with the model group $(\mathrm{P}<0.05)$. The intervention of Xiaochaihu Decoction can change the state of abnormally elevated IL-17, CCL2 and MAOA expression levels.

Conclusions The therapeutic efficacy remarkable from the theory of heating into the sperm chamber to experimental autoimmune prostatitis rats.

Key words Xiaochaihu Decoction; experimental autoimmune prostatitis; cytokines; MAOA

\section{INTRODUCTION}

Chronic prostatitis/chronic pelvic pain syndrome (CP/CPPS) is a commonly observed distress in male patients which is characterized by chronic pelvic discomfort, with voiding symptoms, sexual dysfunction, and associated mental health disorders (MHD)1. Though current antibiotic, alpha-blocker, and electro-magnetic therapy seems promising, the efficient treatment of CP/CPPS remains rare2. Traditional Chinese Medicine (TCM) has therapeutic effects on the disease, but as there is little experimental research and the therapeutic 
mechanisms are unclear, TCM popularization and application is hindered. Increasing evidence support that autoimmunity is a major cause of dysfunction of the organs involved in CP/CPPS in both humans and in rodent models of autoimmune CP/CPPS3. Furthermore, inflammatory cytokines play a critical role in pathogenesis of autoimmune dysfunction and prove to be closely correlated to anxiety and depression4. Thus, we conducted this study to evaluate the effect of Xiaochaihu Decoction on cytokines and MAO in EAP rats. These results give evidence for the drug mechanism of action and provide a theoretical basis for the selection of TCM therapeutic.

\section{MATERIALS AND METHODS}

\section{Experimental animals}

Specific pathogen-free (SPF) Wistar rats were procured from the experimental animal center of Shandong University (Jinan, China). Rats were housed in an SPF environment in a standard housing room under controlled temperature $\left(15-25^{\circ} \mathrm{C}\right)$, relative humidity $(50-70 \%)$, and artificial light ( $12 \mathrm{~h} \mathrm{light/dark} \mathrm{cycle),} \mathrm{and}$ were provided free access to food and water. All animal protocols were approved by the ethics committee of The Qilu Hospital of Shandong University (Jinan, China).

\section{Preparation of rat prostate protein extractive solution}

Ten male Wistar rats, weighing 350-300g, were intraperitoneally anesthetized under aseptic conditions and a median incision on the lower abdomen was made. The prostate tissue was removed without the adhesive tissue, weighed, and then equal volume of saline containing 0.5\% Triton X-100 (Sigma, Germany) (preliminary high temperature sterilization) was added. The tissue was homogenized with a glass homogenizer in an ice-water bath, and centrifuged at $10000 \mathrm{r} / \mathrm{min}(3000 \times \mathrm{g})$ for $30 \mathrm{~min}$ at $4{ }^{\circ} \mathrm{C}$. The supernatant was removed for detection of protein with the biuret method, 2 and the protein concentration of the extracted solution was diluted to 60 $\mathrm{mg} / \mathrm{mL}$ with PBS buffer.

\section{Animal model and study design}

Adult male Wistar rats (3 months old, weighing 250-300g) were randomly divided into blank group $(\mathrm{n}=10)$, EAP model group $(\mathrm{n}=10)$, and EAP model with Xiaochaihu Decoction treatment group (treatment group) ( $\mathrm{n}$ $=10$ ). Except for the rats in the control group, rats in other groups were modeled respectively on days 0 and $30(60 \mathrm{mg} / \mathrm{mL}), 1 \mathrm{~mL}$ suspension of prostate protein extract and complete Freund's adjuvant (Sigma, Aldrich, United States) was injected subcutaneously, and intraperitoneally injected with diph-theria-pertussis and tetanus vaccine (DPT vaccine). The administration was given by intragastric perfusion (equal concentration method) from the second day (on day 31) after the second injection of modeling, once each day, for 28 days.

\section{Detection of serum cytokines with ELISA}

Blood samples were collected before rat sacrifice and centrifuged $\left(3,000 \mathrm{~g}, 15 \mathrm{~min}, 4^{\circ} \mathrm{C}\right)$ to obtain serum. Serum was stored at $-70^{\circ} \mathrm{C}$ until assayed. IL-6, IL-8, and TNF- $\alpha$ were analyzed by ELISA kits (R\&D Systems, Minneapolis, MN, United States) according to manufacturer's protocols. Serum IL-6, IL-8, and TNF- $\alpha$ levels were determined following measurement of absorbance at $450 \mathrm{~nm}$ using a Bio-Rad microplate reader (BioRad, Hercules, CA, United States).

\section{HE staining of prostate}

The rat's prostate was harvested, washed with $0.15 \mathrm{M}$ saline, fixed in $4 \%$ paraformaldehyde, and embedded in paraffin at $4{ }^{\circ} \mathrm{C}$ overnight. Serial $4 \mu \mathrm{m}$ thick sections were stained with hematoxylin and eosin (HE). After HE staining, pathological changes of the prostatic tissue in each group were observed under light microscopy (Olympus, Japan). 


\section{Quantitative PCR}

TRIzol was used for the extraction of mRNA; tissues specimens were incubated with TRIzol for 5-10 min at room temperature and subsequently centrifuged at $8,263 \times \mathrm{g}$ at $4^{\circ} \mathrm{C}$ for $10 \mathrm{~min}$. cDNA was obtained following reverse transcription; the primer was designed according to sequences; target sequences were amplified using a TIANScript RT kit (cat. no. KR104-02; Tiangen Biotech Co., Ltd., Beijing, China) according to the manufacturer's protocol and the reaction system was constructed for PCR. The housekeeping gene $\beta$-actin was used as the internal control in sample results.

\section{Statistical analysis}

SPSS 19.0 statistical software (SPSS Inc., Chicago, IL, United States) was used for statistical analyses. The data are presented as mean \pm SD and comparisons between groups were performed using one-way ANOVA. A result with $\mathrm{P}<0.05$ was considered statistically significant.

\section{RESULTS}

\section{Pathological changes among the groups}

Pathological changes among the groups In the blank group, the prostate tissues of the rats were intact, and fibers between acinuses and the smooth muscle were normally distributed, with no infiltration of inflammatory cells in the lumen of the gland and mesenchyme. There was also no hyperemia or edema. In the model group, the lumen of the gland in the rats'prostate tissues were dilated and enlarged, with secretion visible in the lumen, and dilated blood vessels with hyperemia. The epithelial cells of the acinus had obvious papillary hyperplasia, the mesenchyme had obvious fibrosis, and there were a great number of lymphocytes and plasma cells. Compared with the model group, after administration of the Xiaochaihu Decoction, the proliferation of epithelial cells was improved, the secretion of the cells was reduced, the inflammation disappeared to varying degrees, and the lumen of the gland was enlarged. Epithelial cells mildly proliferated with a regular arrangement, and there was less secretion in the lumen (Figure 1).

Figure 1 Pathological changes in the groups (HE staining, $\times 100$ )

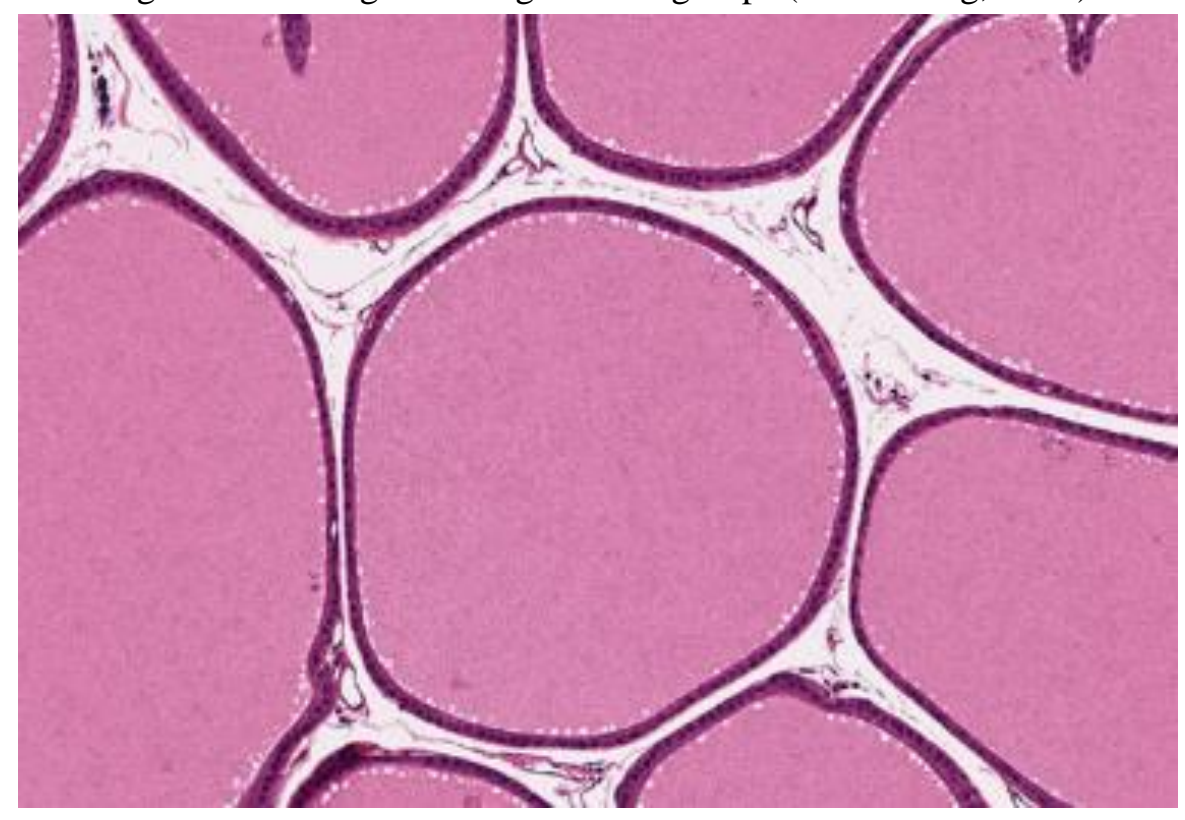

A blank group 
Lu Dongyan, et al. Clinical study of unilateral PVP in the treatment of OVCF in the elderly

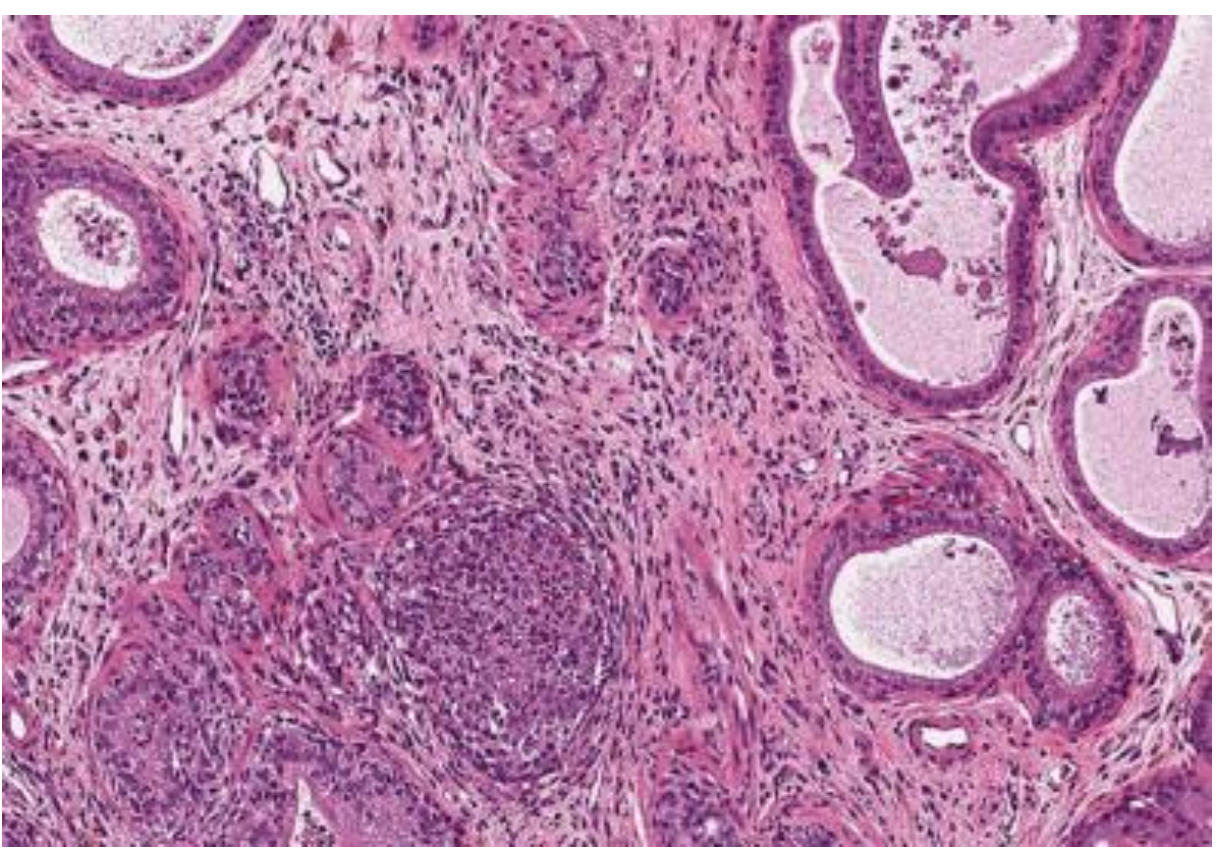

B model group

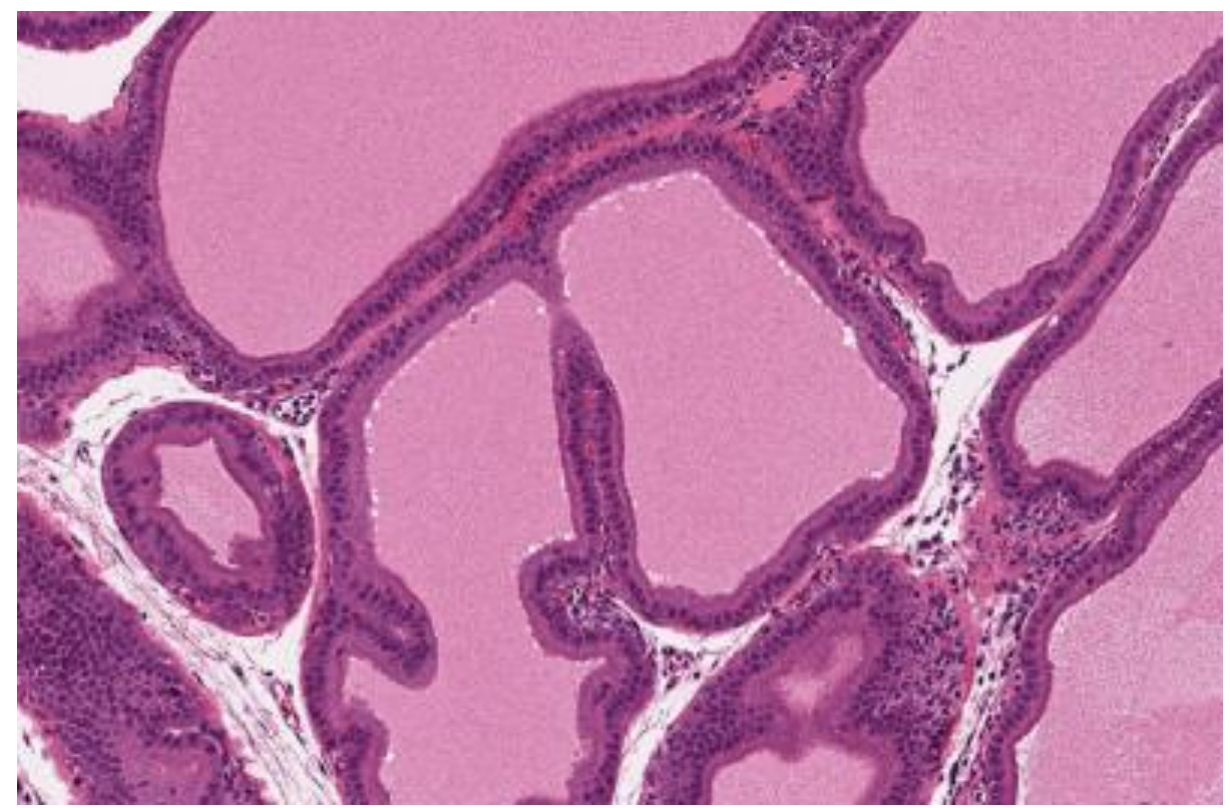

C treatment group

Effects of prescriptions on serum cytokines in the EAP rats

Compared with the blank group, the IL-6, IL-8, and TNF- $\alpha$ levels were significantly higher $(\mathrm{P}<0.05)$ in the model group. After treatment with Xiaochaihu Decoction, the serum IL-6, IL-8, and TNF- $\alpha$ levels all significantly lower in the treatment group as compared with the model group $(\mathrm{P}<0.05)$ (Table 1$)$.

Table 1 Changes in serum IL-6, IL-8, and TNF-a levels

\begin{tabular}{ccccc}
\hline Group & $\mathrm{n}$ & IL-6 $(\mathrm{mol} / \mathrm{l})$ & $\mathrm{IL}-8(\mathrm{pg} / \mathrm{ml})$ & $\mathrm{TNF}-\alpha(\mathrm{pg} / \mathrm{ml})$ \\
\hline blank & 10 & $6.54 \pm 0.11$ & $3.80 \pm 0.60$ & $4.23 \pm 0.43$ \\
model & 10 & $27.52 \pm 2.69^{\triangle}$ & $17.8 \pm 1.43^{\triangle}$ & $18.40 \pm 2.17^{\triangle}$ \\
treatment & 10 & $16.54 \pm 3.24^{\Delta}$ & $4.64 \pm 0.54^{\Delta}$ & $9.22 \pm 1.04^{\Delta}$ \\
\hline
\end{tabular}

Compared with the blank group, ${ }^{\triangle} \mathrm{P}<0.05$, Compared with the model group, ${ }^{\Delta} \mathrm{P}<0.05$.

Effects of prescriptions on prostate tissues cytokines and MAO in the EAP rats 
Wang Zhiqiang, et al. Effect of Xiaochaihu Decoction on cytokines and MAO in experimental autoimmune prostatitis rats Medical Research ISSN 2664-0333 eISSN 2664-0341 Volume 2 Issue 4 http://dx.doi.org/10.6913/MRHK.202012_2(4).0002

IL-17, CCL2 and MAOA/B exhibited high expression in the prostate tissues of EAP rats, indicating that IL17, CCL2 and MAOA/B serve an important role in the occurrence and development of inflammation. Compared with the model group, the expression of IL-17, CCL2 and MAOA were significantly decreased in the treatment group $(\mathrm{P}<0.05)$. The intervention of Xiaochaihu Decoction can change the state of abnormally elevated IL-17, CCL2 and MAOA expression levels, and there was no significant change in MAOB expression among the groups (Table 2).

Table 2 Changes in prostate tissues IL-17, CCL2 and MAOA/B expression $(n=10)$

\begin{tabular}{ccccc}
\hline Group & IL-17 & CCL2 & MAOA & MAOB \\
\hline blank & $1.04 \pm 0.10$ & $1.08 \pm 0.11$ & $0.761 \pm 0.019$ & $0.562 \pm 0.032$ \\
model & $6.45 \pm 0.67^{\triangle}$ & $11.95 \pm 1.70^{\triangle}$ & $0.786 \pm 0.015^{\triangle}$ & $0.557 \pm 0.026$ \\
treatment & $2.90 \pm 0.13^{\star}$ & $6.57 \pm 0.49^{\wedge}$ & $0.766 \pm 0.011^{\star}$ & $0.564 \pm 0.027$ \\
\hline
\end{tabular}

Compared with the blank group, ${ }^{\wedge} \mathrm{P}<0.05$, Compared with the model group, ${ }^{\wedge} \mathrm{P}<0.05$.

\section{DISCUSSION}

Chronic prostatitis is a disease caused by a number of factors ${ }^{5}$ that may lead to discomfort in the groin area, pelvic pain, irritable urination and sexual dysfunction ${ }^{6}$. Its histological characterization is the infiltration of multinucleate and mononuclear cells into interstitial connective tissues ${ }^{7}$. However, the pathogenesis and diagnostic criteria for chronic prostatitis remains unclear, thus research in this area is hindere ${ }^{8}$. Therefore, appropriate animal models are of great value to understanding and treating this disease ${ }^{9}$. With the development of immunology and molecular biology, the effect of autoimmune factors on morbidity of chronic prostatitis is receiving greater attention ${ }^{10}$. The method of the current study was simple and highly effective; the mortality rate of animals was low and the model was stable, reliable and had good pathology specificity; the pathological changes were similar to the clinical manifestation and the pathogenesis was similar. Therefore, the model may be beneficial in the study of pathogenesis and efficiency for novel treatments for human chronic prostatitis.

Traditional Chinese medicine (TCM) has been used in the treatment of andrological conditions for over a thousand years, and an analysis of more than 5000 evidence-based medical studies provides strong evidence that TCM can be effective in the treatment of chronic prostatitis ${ }^{11}$. In the theory of TCM, diseases are treated according to their symptoms. Thus, chronic prostatitis falls into the category of 'stranguria' and 'turbid semen', which is associated with heating into the sperm chamber. In TCM, the syndrome is characterised by damp and heat (two of the pathogenic factors in TCM), damp heat in the early pathology, blood stasis in the late pathology ${ }^{12}$. The therapeutic regimen therefore focuses on reconciliation Shaoyang, improving symptoms by Xiaochaihu Decoction. The whole prescription clears damp heat to remove external pathogens and improve stagnation, which restores the normal physiologic state of the prostate. In the present study, it was found that after treatment with the prescription for reconciliation Shaoyang, serum IL-6, IL-8, and TNF- $\alpha$ levels were significantly lower than those in the model group. The intervention of Xiaochaihu Decoction can change the state of abnormally elevated IL-17 and CCL2 expression levels in the prostate tissues of EAP rats. This result indicates that the prescription has regulatory action on algogenic and inflammatory factors.

Monoamine oxidase (MAO) is a useful target in the treatment of neurodegenerative diseases and depressive disorders. Both isoforms, MAO-A and MAO-B, are known to play critical roles in disease progression ${ }^{13}$. CP/CPPS frequently show depressive symptoms clinically and increasing evidence indicates a correlation between CP/CPPS and depression. However, the underlying mechanisms of CP/CPPS-related depression remain poorly understood ${ }^{14}$. Here, our experiment found that MAOA/B exhibited high expression in the prostate tissues of EAP rats. The intervention of Xiaochaihu Decoction can change the state of 
Lu Dongyan, et al. Clinical study of unilateral PVP in the treatment of OVCF in the elderly

Medical Research ISSN 2664-0333 eISSN 2664-0341 Volume 2 Issue 4 http://dx.doi.org/10.6913/MRHK.202012_2(4).0002

abnormally elevated MAOA expression levels. These findings provide preclinical validation of xiaochaihu Decoction for therapeutic intent in EAP rats with MHD.

\section{REFERENCES}

1. Krieger JN, Nyberg JrL, Nickel JC. NIH consensus definition and classification of prostatitis. JAMA. 1999 Jul 21;282(3):236-7.

2. Franco JV, Turk T, Jung JH, Xiao YT, Iakhno S, Tirapegui FI, et al. Pharmacological interventions for treating chronic prostatitis/chronic pelvic pain syndrome.Cochrane Database Syst Rev. 2019 Oct 6;10(10):CD012552.

3. Breser ML, Motrich RD, Sanchez LR, Rivero VE. Chronic Pelvic Pain Development and Prostate Inflammation in Strains of Mice With Different Susceptibility to Experimental Autoimmune Prostatitis. Prostate. 2017 Jan;77(1):94-104.

4. Wong L, Done JD, Schaeffer AJ, Thumbikat P. Experimental autoimmune prostatitis induces microglial activation in the spinal cord . Prostate. 2015 Jan;75(1):50-9.

5. Liu X, Fan S, Zheng M, Chen J, Zhang J, Li H. The mediation of interleukin-17 and chemokine ligand 2 in pelvic pain of experimental autoimmune prostatitis. Exp Ther Med. 2017 Jul;14(1):51-58.

6. Birowo P, Rangganata E, Rasyid N, Atmoko W. Efficacy and safety of extracorporeal shockwave therapy for the treatment of chronic non-bacterial prostatitis: A systematic review and meta-analysis. PLoS One. 2020 Dec 28;15(12):e0244295.

7. Ye C, Xiao G, Xu J, Qin S, Luo Y, Chen G, et al. Differential expression of immune factor between patients with chronic prostatitis/chronic pelvic pain syndrome and the healthy volunteers. Int Urol Nephrol. 2018 Mar;50(3):395399.

8. Ho DR, Chang PJ, Lin WY, Huang YC, Lin JH, Huang KT, et al. Beneficial Effects of Inflammatory CytokineTargeting Aptamers in an Animal Model of Chronic Prostatitis. Int J Mol Sci. 2020 May 31;21(11):3953.

9. Shulyak A, Gorpynchenko I, Drannik G, Poroshina T, Savchenko V, The effectiveness of the combination of rectal electrostimulation and an antidepressant in the treatment of chronic abacterial prostatitis. Nurimanov K. Cent European J Urol. 2019;72(1):66-70.

10. Moreira JD, Koch BEV, van Veen S, Walburg KV, Vrieling F, Mara Pinto Dabés Guimarães T, et al. Functional Inhibition of Host Histone Deacetylases (HDACs) Enhances in vitro and in vivo Anti-mycobacterial Activity in Human Macrophages and in Zebrafish. Front Immunol. 2020 Feb 3;11:36.

11. Qiu MX, Xiong GB, Zhou SY, Wang D, Shao JC, Wang JY. Qingrelishi-category Chinese medicine for chronic prostatitis: a systematic review. Zhonghua Nan Ke Xue. 2007;13:370-377.

12. Wang Z, Yuan L, Wang Y, Yang B, Dong X, Gao Z. Efficacy and safety of Chinese herbal medicine for chronic prostatitis associated with damp-heat and blood-stasis syndromes: a meta-analysis and literature review. Patient Prefer Adherence. 2016 Sep 19;10:1889-1902.

13. Gaur S, Gross ME, Liao CP, Qian B, Shih JC. Effect of Monoamine oxidase A (MAOA) inhibitors on androgensensitive and castration-resistant prostate cancer cells. Prostate. 2019 May;79(6):667-677.

14. Du HX, Chen XG, Zhang L, Liu Y, Zhan CS, Chen J, et al. Microglial activation and neurobiological alterations in experimental autoimmune prostatitis-induced depressive-like behavior in mice. Neuropsychiatr Dis Treat. 2019 Aug 6;15:2231-2245. 